\title{
Application of wavelet spectral analysis to plasma fluctuation measurements using beam emission spectroscopy
}

\author{
M. Jakubowski, R. Fonck, J. S. Kim, and G. McKee \\ Department of Engineering Physics, University of Wisconsin, Madison, Wisconsin 53706
}

(Presented on 10 June 1998)

\begin{abstract}
Beam emission spectroscopy (BES) measures localized density fluctuations in fusion plasma turbulence. A wavelet transform method of spectral analysis is applied to BES data to extract fluctuations without the long time-averaging characteristic of Fourier transform spectral analysis methods. This technique is used to observe an abrupt $(\sim 100 \mu \mathrm{s}) L-H$ transition on DIII-D. Wavelet-based cross correlation analysis can be used to obtain fluctuations of poloidal rotation velocity, and suggests opportunity for measurement of electrostatic potential fluctuation measurements. (C) 1999 American Institute of Physics. [S0034-6748(99)64901-2]
\end{abstract}

\section{INTRODUCTION}

Turbulence in magnetically confined plasmas continues to be one of the most important scientifically challenging research topics in controlled thermonuclear fusion research. Turbulence manifests itself as fluctuations in plasma parameters such as the density, temperature, and electrostatic potential. Beam emission spectroscopy (BES) was created to measure long-wavelength $(k \rho<1)$ density fluctuations in the core and edge regions of tokamak plasmas, and has produced unique observations on the TFTR, ${ }^{1}$ TEXT, ${ }^{2}$ and DIII-D ${ }^{3}$ experiments.

The BES experiment on DIII-D presently measures density fluctuations at 32 spatial locations spanning a cross section of the fusion tokamak to gain insight into both microand macroturbulence phenomena. ${ }^{4}$

To date, the reduction and interpretation of data from BES measurements have relied on Fourier transform spectral analysis techniques. Applications of these techniques have produced information on a wide range of turbulence phenomena in tokamaks, including radial profiles of turbulence amplitudes, ${ }^{5}$ wave number spectra, ${ }^{1}$ turbulence correlation lengths, ${ }^{1}$ linear growth rates, ${ }^{6}$ localized edge to core transitions, ${ }^{7}$ existence of improved confinement regime transition dynamics, ${ }^{3}$ magnetic island structures, ${ }^{5}$ characterization of toroidal Alfvén eigenmodes, ${ }^{8}$ and disruption precursors, among others.

The use of Fourier spectral analysis is most applicable to studies of stationary turbulence, and it imposes conditions on the achievable time resolution of turbulence measurements due to the need for repeated measurements across sequential time bins to achieve a requisite signal-to-noise level in the power spectrum. In addition, there is a need to obtain a precise measure of the photon statistical noise inherent in BES measurements using Fourier analysis techniques. Both of these phenomena limited past measurement using BES to moderate to poor temporal resolution (i.e., $\sim 10-500 \mathrm{~ms}$ ).

This, in turn, has limited the ability to study interesting transient or intermittent phenomena in plasma turbulence in high temperature tokamaks. Several topics in current hightemperature plasma science involve transient variations in local plasma turbulence and could benefit from measurements providing the unique abilities of BES, such as good spatial localization, long wavelength sensitivity, and simultaneous radial and poloidal resolution. Representative transient phenomena which are of special interest include the transition from low $(L)$ to high $(H)$ confinement regimes, formation of internal transport barriers, ${ }^{3}$ and intermittent bursting magnetohydrodynamic (MHD)-like fluctuations. ${ }^{9}$

In this article, we discuss the use of wavelet spectral analysis techniques to provide high time-resolution measurements of turbulence with BES. The ability of wavelets to provide good time resolution with modest frequency resolution matches well with the character of the broadband spectra typical of BES data. In general, both the Fourier and wavelet transforms fall in the more general category of adaptive wave form analysis techniques, where the Fourier approach is adapted to long lived, stationary signals, while wavelet transforms are more adapted to the study of transient or intermittent behavior.

In the next section, we introduce a specific wavelet transform as applied initially to BES data analysis. This is followed by application to specific transient changes observed in tokamak plasma turbulence. We then discuss a particularly interesting and novel application of wavelet transforms to the study of the rotation-induced time lag between two adjacent BES channels separated in the poloidal direction by a distance less than the decorrelation length. The use of wavelet transforms to extract the time evolution of this time lag presents a direct measure of transient changes in the plasma rotation speeds, and could eventually lead to measurement of local electrostatic potential fluctuations using BES.

\section{WAVELET TRANSFORM FOR BES SPECTRAL ANALYSIS}

Wavelets have the property of being time localized and frequency scale selectable, and as such are capable of high time resolution. Wavelets extract both time evolution and frequency composition of a signal, while Fourier sines extract only frequency information from a time signal, thus 


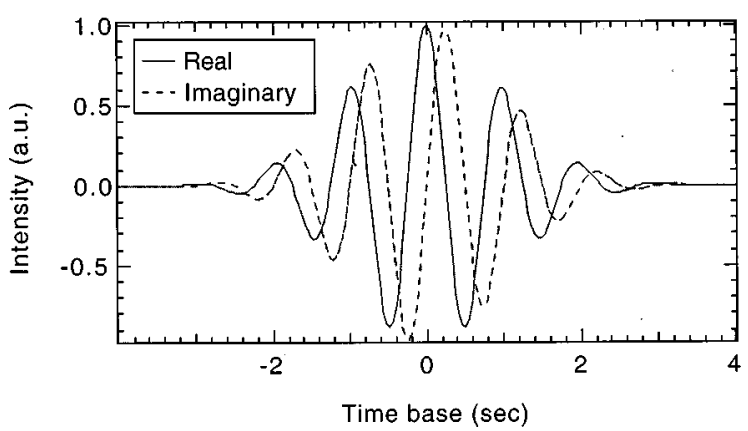

FIG. 1. Morlet wavelet, real and imaginary components.

losing time information. The reader is referred to the abundant tutorial literature on the subject of wavelets for details. ${ }^{10}$

For this initial application of wavelets to BES analysis, we focus on the particular wavelet basis used, the Morlet wavelet: ${ }^{11}$

$$
\psi(t)=e^{i \omega_{0} t} e^{-t^{2} / 2},
$$

shown in Fig. 1. The Morlet wavelet transform is defined as

$$
W(a, \tau)=\int_{-\infty}^{+\infty} f(t) \psi_{\mathrm{a} \tau}^{*} d t .
$$

It should be noted that $\psi$ defined in Eq. (1) is the mother wavelet, while $\psi_{\mathrm{a} \tau}$ are translations and dilations of the original wavelet defined by

$$
\psi_{\mathrm{a} \tau}=\frac{1}{\sqrt{a}} \psi\left(\frac{t-\tau}{a}\right) .
$$

The wavelet transform scans the signal with both frequency scale dilations and time space translations (entire wavelet "slides" along the time axis) of the mother wavelet to convert the initial temporal signal into a more redundant representation in time and frequency. One may thus see that scaling contraction of the wavelet ("squeezing" the wavelet) leads to high temporal localization, the essence of its attractiveness for our purposes. Moreover, it should be said that the broadband frequency nature of the turbulent signals seen in BES ends itself further to high temporal resolution due to the $\Delta f \Delta t \leqslant 1$ relation between the width of the temporal distribution and its frequency transform.

The complex wavelet auto- and cross-power spectra are defined as

$$
W_{f g}(a, \tau)=W_{f}^{*}(a, \tau) W_{g}(a, \tau)
$$

with $f=g$ and $f \neq g$, respectively. Moreover, the cross phase (to be used in rotation velocity fluctuations) is given by: ${ }^{12}$

$$
\Theta(a, \tau)=\tan ^{-1} \frac{\operatorname{im}[W(a, \tau)]}{\operatorname{re}[W(a, \tau)]} .
$$

\section{OBSERVATION OF TRANSIENT CHANGES IN TURBULENCE VIA BES}

The time resolution provided by wavelet transform analysis allows the observation of sudden changes in the turbulence level associated with the low-to-high $(L-H)$ con-

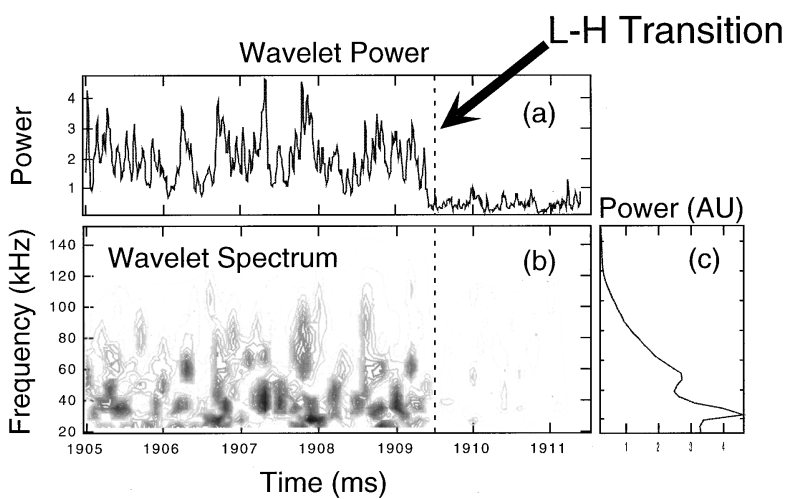

FIG. 2. (a) The evolution of turbulence power summed over frequency in a $L-H$ transition on DIIID as derived via wavelet transform, where the magnitude of the turbulence drops suddenly by at least a factor of 5 within a period of $\leqslant 100 \mu$ s. (b) Contour plot of the derived wavelet power as a function of time and frequency (c) power (summed over time) as a function of frequency (vertical axis).

finement mode transition on the DIII-D tokamak. Sample results of turbulence behavior a few centimeters inside the separatrix on DIII-D are shown in Fig. 2. The true evolution of the turbulence power as derived via wavelet transform is shown in Fig. 2(a), while Fig. 2(b) shows the derived frequency spectrum as a function of time. Figure 2(c) shows the power summed over time as a function of frequency (vertical axis). Note that Fig. 2(c) corresponds to Fourier transform spectral analysis, where power is expressed as a function of frequency. Information found in typical Fourier analysis is contained in Fig. 2(c), in that we see a sharp, low frequency peak around $20 \mathrm{kHz}$ corresponding to MHD density fluctuations, and we observe the broadband microturbulence from $50 \mathrm{kHz}$ and up, fading out at higher frequencies.

The time evolution of the fluctuating power in Fig. 2(a) illustrates the improved time resolution of BES measurements afforded by the use of the wavelet transform. It is seen clearly in Fig. 2(a) that there is a sudden drop in the magnitude of the turbulence by at least a factor of 5 within a period of $\leqslant 100 \mu$ s, centered at $1909.4 \mathrm{~ms}$.

While such a sharp reduction in the turbulence power is noticeable in the raw BES data, the wavelet-transformed power provides better discrimination against background noise, frequency band isolation and a clear, rapid drop in fluctuations. Rapid $L-H$ transition of turbulence levels has also been documented with the far-infrared scattering (FIR) diagnostic ${ }^{13}$ and edge probes. ${ }^{14}$

Wavelet spectral analysis has also been applied to study the transient behavior of BES density fluctuations of a reversed shear (RS) confinement mode on TFTR. High time resolution analysis of the RS mode shows consistent $\sim 100$ $\mu$ s MHD bursting behavior. In addition, wavelet analysis has been applied to Langmuir probe data to detect intermittence of structure in wave-wave coupling on $500 \mu$ s time scales on the ATF stellarator by other groups at Oak Ridge National Laboratory. ${ }^{11}$ 


\section{TIME EVOLUTION OF POLOIDAL PLASMA FLOWS VIA TIME-VARYING CROSS PHASE}

The use of wavelet transforms for cross correlations provides the ability to measure rapid variations in the plasma poloidal $E \times B$ velocity. For two adjacent BES channels, wavelet analysis allows us to track a time-varying time lag, $\tau(t)$, through the time-varying cross-phase $\Delta \phi(t)$ :

$$
\tau(t)=\Delta \phi(t) / 2 \pi f,
$$

where $f$ is the frequency. The time-varying time lag is directly related to the time-varying poloidal rotation velocity

$$
\tau(t)=\Delta z / v_{\theta}(t)
$$

where $\Delta z$ is the BES spatial channel separation in the poloidal direction. Under the assumption that diamagnetic flows are small compared to rotation speeds, as is typical for DIII-D plasmas $\left(v_{1}^{*}<0.1 v_{E \times B}\right)$, the apparent poloidal speed of the fluctuations in the laboratory frame is given by the radial electric field $\left(E_{r}\right)$ as

$$
v_{\theta}(t)=E_{r}(t) / B_{\phi} .
$$

In general, BES fluctuation spectra are dominated by this rotation-induced Doppler shift, and hence show a linear dependence between the cross phase and frequency. Hence, $\tau(t)$ is independent of frequency. The use of wavelet transform to derive $\tau(t)$ and hence $v_{\theta}(t)$ gives a means of observing rapid variations in plasma flow speeds. At moderate time resolution, this can provide significant data on the formation of internal transport barriers and their relation to the local flow velocity shear. If the poloidal rotation velocity fluctuation can be obtained on plasma turbulence-relevant scales (few $\mu \mathrm{s}$ ), we then obtain the fluctuating radial electric field and thus electrostatic potential on time scales relevant to transport. This potentially provides measurements of another crucial element in estimating the turbulence-driven anomalous cross-field particle transport in tokamaks, given by

$$
\Gamma_{\text {anom }}=\left\langle\tilde{n} \tilde{v}_{r}\right\rangle \propto\langle\tilde{n} \tilde{\Phi}\rangle .
$$

To that end, we examine the ability of cross correlation via wavelet transforms to extract time variations in the lag time between poloidally adjacent BES sample volumes.

\section{A. Sensitivity analysis for extraction of variable lag times between correlated samples}

To evaluate the effectiveness of wavelet analysis in extracting time-varying correlation lag times, we have conducted initial tests using simulated fluctuation data. We used both individual sine waves and simulated noise free as well as noisy turbulence. Initial results show effective fluctuating phase extraction.

\section{Sine waves}

Through the cross phase, Eq. (5), wavelet analysis can extract a time-varying phase between two signals consisting of a simple sine wave. Figure 3 shows the results from a simulation with two time signals having a well-defined frequency and an imposed relative oscillatory phase shift in the second half of the time trace. The input signals are defined as
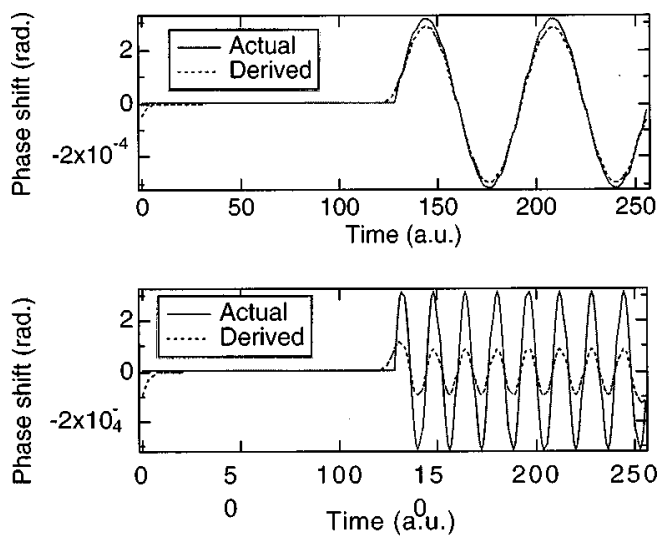

FIG. 3. Phase shift in simulated data derived with wavelet technique, along with actual imposed phase shift: (a) low frequency phase shift and (b) high frequency phase shift.

$$
\begin{aligned}
& S_{1}=S_{0} \sin (\omega t) \\
& S_{2}=S_{0} \sin \left[\omega t+A_{0} \sin (\theta t)\right] .
\end{aligned}
$$

Note that it is the phase difference $\left[A_{0} \sin (\theta t)\right]$ that is being recovered using the Morlet wavelet basis from the wavelet cross-power spectrum, Eq. (4), according to Eq. (5), and that the phase difference is imposed only on the second half of the signal in order to observe the effects of an onset. For sufficiently "slow" oscillating phase shifts, the instantaneous phase shift or time lag is readily recovered with the method, as shown in Fig. 3.

This test indicates that as time resolution is pushed to the digital sampling time, the sensitivity to oscillating phase differences is reduced. This will place a minimum time resolution as turbulence-relevant time scales are approached. Furthermore, note that in both graphs in Fig. 3, the phase starts to rise $\sim 10$ time points before the actual phase difference is imposed, which shows that the Morlet wavelet does not have perfect (delta function) time localization. We expect that by optimizing the wavelet basis and using broadband simulated data that mimics actual fluctuation data more closely, and possibly increasing the data sampling rate, the minimum time resolution can be reduced to transport-relevant time scales.

\section{Simulated turbulence with variable lag time}

The cross-phase evaluation [Eq. (5)] was applied to the wavelet cross-power spectrum (4) of two distinct turbulence signals with frequencies defined by a Gaussian amplitude profile and random phase (distinct for each signal) [Fig. 4(a)]. A coherent oscillating phase shift was added to the second turbulence signal, and the resultant data pair analyzed via Eq. (5) to extract the time-varying cross phase.

We observe clear temporal phase reconstruction [Eq. (5)] in Fig. 4(b), where the broken line shows the imposed phase and the solid line shows the cross phase derived via the wavelet transform. As the frequency of the oscillating phase imposed on the second signal increases, the phase correlation to the reconstructed signal decreases, as shown in Fig. 4(c). The correlation coefficient of imposed to recon- 

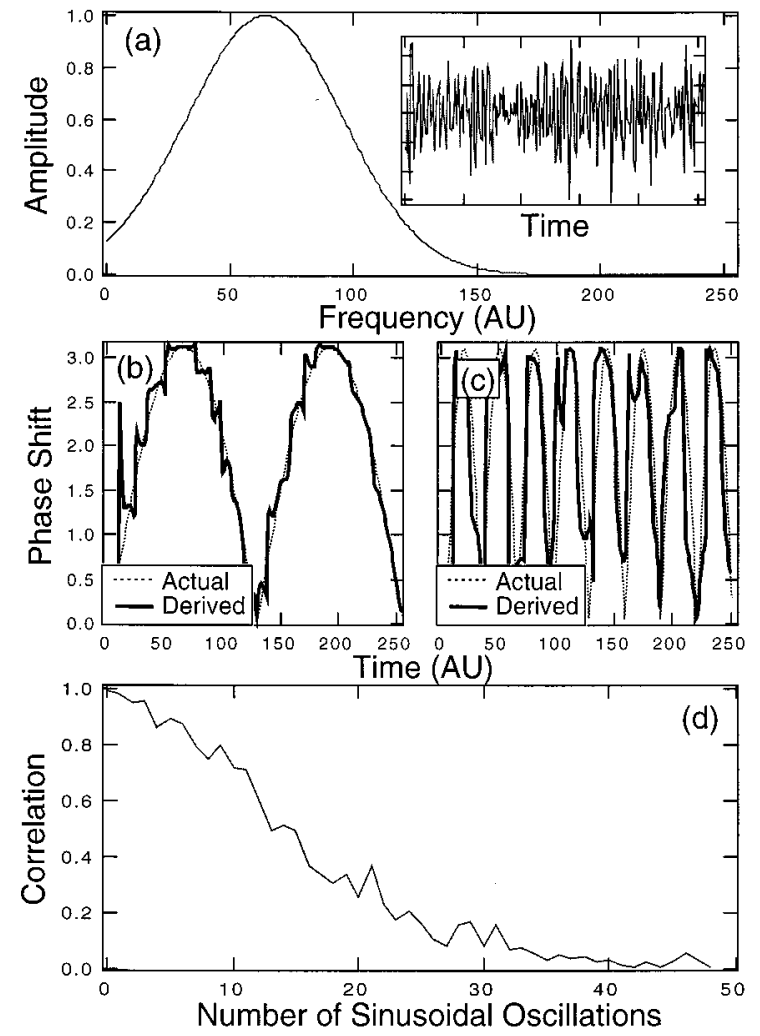

FIG. 4. (a) Simulated turbulent signal in frequency and time used in the wavelet method of cross-phase extraction; (b) phase tracking in time for one full sinusoidal oscillation of phase; (c) phase tracking for four full oscillations; (d) correlation coefficient of phase tracking as a function of the number of full sinusoidal oscillations of phase.

structed phase is shown in Fig. 4(d) as a function of the number of full sinusoidal oscillations of imposed phase.

To test the extractability of the cross phase when the signal is polluted with noise, we added white noise to the simulation, which manifests itself as a dc offset in the frequency space representation of the turbulent signal and is similar to actual photon noise obtained in BES data. The correlation of imposed phase with its reconstruction is shown in Fig. 5(c) as a function of the noise level. It shows that when the noise level was a factor of $\sim 50$ times as large as the signal itself, the wavelet method of phase extraction showed at least a hint of the imposed phase shift. Therefore, when the magnitude of white (photon) noise is similar to the magnitude of the turbulence signal, the results have a correlation near 1 [Fig. 5(b)].

\section{SUMMARY}

The wavelet transform provides a spectral analysis tool which allows the extraction of the time evolution of turbulence characteristics measured via BES. As such, it extends the measurement capabilities of the BES diagnostic technique to time scales of $\leqslant 100 \mu \mathrm{s}$. Through wavelet-based cross-correlation techniques, it provides measurement of the particularly interesting plasma rotation velocity fluctuations,

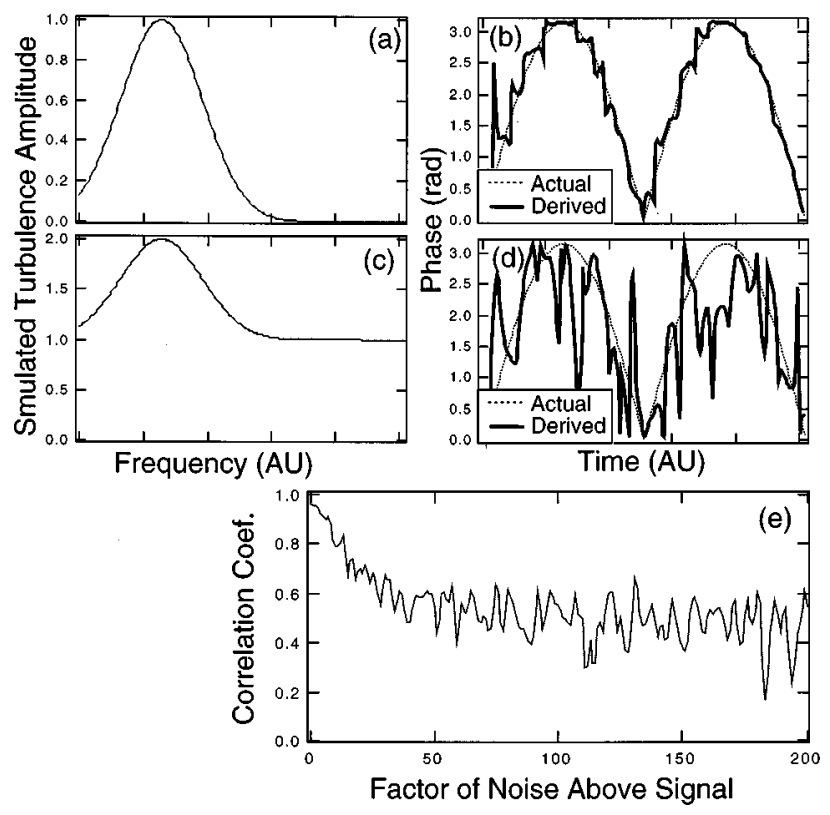

FIG. 5. Tracking of an oscillating phase via the wavelet method. (a) Pure turbulence in frequency space $\left(a^{\prime}\right)$; phase tracking of pure turbulence; (b) turbulence tracking within turbulence hidden in an equal magnitude of white noise; (c) correlation coefficient of imposed phase oscillation to wavelet method-tracked phase oscillation as a function of the factor of noise above the signal.

and may eventually lead to measurements of electrostatic potential fluctuations if sufficiently high time resolution can be obtained with acceptable signal-to-noise.

\section{ACKNOWLEDGMENTS}

The authors would like to thank Guido Buresti at University of Pisa, Italy, for initial discussion of the temporal phase extraction method. We would also like to thank Curt Rettig at General Atomics for sharing FIR scattering data for $L-H$ transition dynamics comparison. This work was supported by U.S. Department of Energy Grant No. DE-FG0289ER53296.

${ }^{1}$ R. J. Fonck et al., Phys. Rev. Lett. 70, 3736 (1993).

${ }^{2}$ R. D. Durst et al., Rev. Sci. Instrum. 66, 842 (1995).

${ }^{3}$ G. R. McKee et al., Bull. Am. Phys. Soc. 42, 1921 (1997).

${ }^{4}$ G. R. McKee et al., Rev. Sci. Instrum. (these proceedings).

${ }^{5}$ M. Jakubowski et al., Bull. Am. Phys. Soc. 42, 1921 (1997).

${ }^{6}$ J. S. Kim, Thesis, University of Wisconsin, Madison, 1996.

${ }^{7}$ R. D. Durst et al., Phys. Rev. Lett. 71, 3135 (1993).

${ }^{8}$ R. D. Durst and R. J. Fonck, Phys. Fluids B 4, 3707 (1992).

${ }^{9}$ J. S. Kim (unpublished).

${ }^{10}$ I. Daubechies, Ten Lectures on Wavelets (American Mathematical Society, Providence, 1992); C. Chui, An Introduction to Wavelets (Academic, New York, 1992); D. E. Newland, An Introduction to Random Vibrations, Spectral and Wavelet Analysis (Longman Scientific \& Technical, New York, 1993); L. Hudgins et al., Phys. Rev. Lett. 71, 3279 (1993); R. R. Coifman and M. V. Wickerhauser, Proceedings of Symposia in Applied Mathematics, edited by I. Daubichies (American Mathematical Society, Providence, 1993), Vol. 47, p. 119.

${ }^{11}$ B. P. van Millagen, C. Hidalgo, and E. Sanchez, Phys. Rev. Lett. 74, 395 (1995).

${ }^{12}$ M. Onorato et al., Eur. J. Mech. B/Fluids 16, 575 (1997).

${ }^{13}$ C. L. Rettig et al., Phys. Plasmas 4, 4009 (1997).

${ }^{14}$ R. A. Moyer et al., Phys. Plasmas 2, 2397 (1995). 